\title{
Cloning and Characterization of Disease Resistance Protein RPM1 Genes against Powdery Mildew in Wheat Line N9134
}

\author{
Y.B. $\mathrm{NIE}^{1,2}$ and W.Q. JI ${ }^{1 *}$ \\ ${ }^{1}$ State Key Laboratory of Crop Stress Biology for Arid Areas, College of Agronomy \\ (Northwest A\&F University), Yangling, Shaanxi 712100, China \\ ${ }^{2}$ Institute of Crop Research, Xinjiang Academy of Agri-Reclamation Sciences, Shihezi, \\ Xinjiang 832000, China \\ (Accepted 16 April 2018; Received 30 April 2019; \\ Communicated by A. Mohan)
}

\begin{abstract}
Powdery mildew (Blumeria graminis f. sp. tritici) is one of the most devastating wheat diseases. The wheat line N9134 contains PmAS846 that was transferred to N9134 from wild emmer wheat, and is still one of the most effective resistance genes in China. A full-length wheat RPM1 gene was obtained by rapid amplification of cDNA ends (RACE) based on the up-regulated probe sequence from differentially expressed transcripts during the N9134 and powdery mildew interaction. The gene was named TaRPMI, and the open reading frame (ORF) is 2721 nucleotides and encodes a polypeptide of 907 amino acids with a predicted isoelectric point of 4.86. Phylogenetic analysis revealed that TaRPM1 was highly homologous on both Aegilops tauschii and Triticum urartu at both the nucleotide and protein level. Using real-time quantitative PCR, the TaRPM1 gene expression level in wheat leaves was found to be sharply up-regulated, while the transcript level was lowly induced in the root and stem. Under the powdery mildew treatment, the transcription profile of TaRPM1 was very strongly expressed at 48 hour post inoculation (hpi), which increased again to $96 \mathrm{hpi}$ and reaching a high level at $120 \mathrm{hpi}$. Based on sequence similarities and positions, we inferred that the TaRPM1 gene was on wheat chromosome 3D. These results suggested that TaRPM1 plays an important role in the mechanism of innate immunity to infection by the powdery mildew pathogen.
\end{abstract}

Keywords: gene clone, sequence characterization, expression analysis, resistance protein

\section{Introduction}

Wheat powdery mildew, caused by Blumeria graminis f. sp. tritici $(B g t)$, is one of the most destructive diseases of wheat worldwide. Genetic resistance is the most economical and effective method to control this disease. To date, 69 powdery mildew resistance alleles have been identified and designated at 49 loci. Wheat is a hexaploid crop with a tremendous genome of 16,000 Mb (Appels et al. 2018), which contains three sub-genomes (A, B and C) with a large number of repeat sequences. Thus far, few wheat powdery mildew resistance genes (Pm21, Pm3b, Pm 3c, Pm3f, and Pm3g) have been cloned (Cao et al. 2011; Yahiaoui et al. 2004; Srichumpa et al. 2005; Tommasini et al. 2006). 
RPM1 was one of the first plant resistance genes to be identified from natural variation in Arabidopsis thaliana (Grant et al. 1995). Through analysis of conservative sequences, research has shown that resistance $(\mathrm{R})$ genes in plants share conserved regions, including leucine-rich repeat (LRR), nucleotide-binding site (NBS), transmembrane domain, protein kinases and serine/threonine kinase regions (Collins et al. 2001; Swiderski et al. 2009).

Among the wheat R genes, the Sr35 and Sr33 genes have an NBS-LRR resistance domain (Periyannan et al. 2013; Saintenac et al. 2013). However, the nucleotide-binding sites and leucine-rich repeat domains, as well as variable amino domains (Gong et al. 2013; McHale et al. 2006), of NBS-LRR genes may have evolved rapidly in bread wheat (Gu et al. 2015). The role of the RPMI gene in wheat with respect to resistance to powdery mildew is not fully understood.

The wheat line N9134 contains a powdery mildew resistance gene, PmAS846, that was introgressed into common wheat from the wild emmer accession As846 (Xue et al. 2012). PmAS846 provides a potent resistance that is effective against 21 Chinese Bgt isolates with different virulence patterns (Wang et al. 2007), and should be a valuable resource in wheat breeding and research programs. Previously, the gene was physically located on wheat chromosome 5BL bin 0.75-0.76 (Xue et al. 2012). The wheat line N9134 inoculated powdery mildew at $12 \mathrm{~h}, 24 \mathrm{~h}$, and $48 \mathrm{~h}$ was analyzed by gene chip (Illumina). The results showed that thirty probes were differentially expressed, with sequences showing a NBS, LRR, and serine/threonine protein kinases domain.

In the present study, we identified a fragment of the $R P M 1$ gene (GenBank accession number: AJ606014.1) that was up-regulated by powdery mildew infection. We obtained the full-length sequence of the corresponding wheat gene named TaRPM1 using the rapid amplification of cDNA ends (RACE), delineating its expression profile in the presence of powdery mildew infection and various stress treatments. The results of these studies indicate that the activation of TaRPM1 may be a general response to powdery mildew infection.

\section{Materials and Methods}

\section{Plant materials and treatments}

Winter wheat (Triticum aestivum L.) genotype N9134 and powdery mildew race E09 were used in this study for the full-length cloning of the wheat TaRPM1 gene and its expression analysis. The Bgt isolate E09 was maintained on susceptible wheat 'Shaanyou $225^{\prime}$. The germinated seedlings were grown in sterilized vermiculite and cultured with a cycle of $12 \mathrm{~h}$-artificial light at $22{ }^{\circ} \mathrm{C}$ and $12 \mathrm{~h}$ dark at $14{ }^{\circ} \mathrm{C}$ with $70 \%$ relative humidity. At the three-leaf development stage, twenty-four plants of each genotype were inoculated with E09. Talc was used to aid in the spread and adhesion of spores over leaf surfaces. Eight strains of each control flats were treated the same way except for the absence of spores in the talc. 
In order to perform tissue-specific expression analyses of TaRPM1, intact root, stem, and leaf tissues of 3-week-old wheat seedlings were collected from inoculated plants. All samples in these experiments were collected, quickly frozen in liquid nitrogen and stored at $-80{ }^{\circ} \mathrm{C}$ prior to RNA extraction. Three independent biological replications were performed for each treatment.

\section{RNA isolation and purification}

Total RNA was extracted independently for each individual leaf sample using Trizol reagent according to the manufacturer's instructions (Invitrogen Corporation). The RNA was purified using the RNeasy Kit (Takara) according to the manufacturer's instructions. RNA quality and quantity were assessed using a spectrophotometer and agarose gel electrophoresis. The RNA acceptable purity was indicated by A260:280 ratios of 1.9-2.1 in $10 \mathrm{mM}$ Tris- $\mathrm{HCl}(\mathrm{pH} 7.5)$.

\section{Rapid amplification of cDNA ends (RACE)}

The up-regulated probe Ta.30657.1.S1 at (GenBank accession No. AJ606014.1) was a candidate gene of 574 base pairs (bp) in length, encoding the Triticum aestivum partial mRNA for putative NB-ARC protein. To obtain a full-length cDNA of the wheat TaRPMI gene, a pair of gene-specific primers (forward primer: 5'-ATGGATGCTCGTCGTCTGTC-3', reverse primer: 5'-GGGAGTGACCTGACCCTAGT-3') was designed based on the AJ606014.1 assembly sequence and synthesized (Shanghai GeneCore BioTechnologies Co., Ltd.).

\section{Sequence and phylogenetic analysis}

The cDNA sequence data was analyzed using BLAST (https://blast.ncbi.nlm.nih.gov/ Blast.cgi), the CAP3 Sequence Assembly Program (http://doua.prabi.fr/software/cap3), ORF Finder (https://www.ncbi.nlm.nih.gov/orffinder/), VecScreen (https://www.ncbi. nlm.nih.gov/tools/vecscreen/), and PROSITE (https://prosite.expasy.org/) network services. The signal peptide was predicted using TargetIP (http://www.cbs.dtu.dk/services/ SignalP/). Transmembrane analysis was performed using TMPRED (https:/embnet.vitalit.ch/software/TMPRED_form.html). The alignments of the deduced protein sequences and phylogenetic trees were computed using the MEGA software version 7.0 programs, respectively, employing standard parameters.

\section{Expression analysis of RPM1 gene in different tissues}

Use qRT-PCR to detect the specific expression of TaRPM1 in wheat different tissues, including roots, stems and leaves of wheat new germplasm N9134 after inoculation with powdery mildew E09 at $48 \mathrm{~h}$. 
Use the Expression Atlas website (https://www.ebi.ac.uk/gxa/genes/TraesCS3B02G48 3600 ?bs $=\% 7 \mathrm{~B} \% 22$ triticum $\% 20$ aestivum $\% 22 \% 3 \mathrm{~A} \% 5 \mathrm{~B} \% 22$ ORGANISM PART $\% 22 \% 2 \mathrm{C} \% 22$ DEVELOPMENTAL_STAGE $\% 22 \% 2 \mathrm{C} \% 22 \mathrm{SAMPLING} T \mathrm{TIME}$ POINT\%22\%5D\%7D\&ds=\%7B\%22kingdom \%22\%3A\%5B \%22plants $\% 22 \%$ $5 \mathrm{D} \% 7 \mathrm{D} \#$ baseline) further verified the tissue-specific expression analysis of RPM1 gene.

\section{Real time-PCR analysis}

Expression profile of TaRPMI after inoculation with $B g t$ was detected by qRT-PCR analysis with a pair of specific primers Q-RPM1-FP and Q-RPM1-RP (RPM1-FP: GGGAGGACACGAAAAGAGCAA; RPM1-RP: GGCTTCAAGGATGACTGGGAT). For qRTPCR, SYBR ${ }^{\circledR}$ Premix DimerEraser ${ }^{\mathrm{TM}}(\mathrm{TaKaRa})$ was used according to the manufacturer's protocols and analyzed using an ABI 7300 RT-PCR system (Applied Biosystems). The qRT-PCR amplification mixture $(25 \mu \mathrm{L})$ contained $2 \mu \mathrm{L} 20 \times$ first-strands cDNA, $12.5 \mu \mathrm{L}$ of SYBR Green PCR Master Mix, and $2 \mu \mathrm{L}$ of forward and reverse primers $(10 \mu \mathrm{M})$. The wheat tubulin gene (GenBank accession No. Y049040) was used as a control for normalization. Three independent experiments were performed, including three non-template controls. Each $25 \mu \mathrm{l}$ reaction was denatured at $95{ }^{\circ} \mathrm{C}$ with an initial denaturation step $\left(95^{\circ} \mathrm{C} / 2 \mathrm{~min}\right)$, followed by 40 cycles of $95^{\circ} \mathrm{C} / 10 \mathrm{~s}, 60^{\circ} \mathrm{C} / 20 \mathrm{~s}$, and $72{ }^{\circ} \mathrm{C} / 20 \mathrm{~s}$. A melting curve analysis was performed over the range $80-95^{\circ} \mathrm{C}$ at $0.5^{\circ} \mathrm{C}$ intervals. Dissociation curves were generated for each reaction to ensure specific amplification. The transcript concentration was calculated as $2^{-\Delta \Delta \mathrm{Ct}}$. To avoid errors caused by operational factors, and each data point was expressed as the average \pm SD of three independent biological replicates.

\section{Chromosome localization prediction of TaRPM1}

The gene sequence of TaRPM1 was compared with the genome of Chinese spring wheat (http://plants.ensembl.org/Triticum_aestivum/Info/Index?tdsourcetag=s_pcqq_aiomsg). The structure of TaRPM1 analyzed using GSDS 2.0 (Gene Structure Display Server) version for intron and exon prediction. A highly consistent sequence was screened and the target gene was mapped to the wheat chromosome (Qiao et al. 2016).

\section{Results}

\section{Isolation and sequence analysis of TaRPM1 cDNA}

The probe Ta.30657.1.S1_at (AJ606014.1) was an infection-induced up-regulated gene fragment identified in a previous experiment using gene chips (Xue 2012). Following 3' and 5' RACE analyses, two cDNA fragments of approximately 1246 (Fig. 2) and 944 bp (Fig. 1), respectively, were detected on 1\% agarose gel. The results showed the full-length sequence of wheat $R P M 1$, which consisted of a 3034 nucleotide and cDNA containing an 


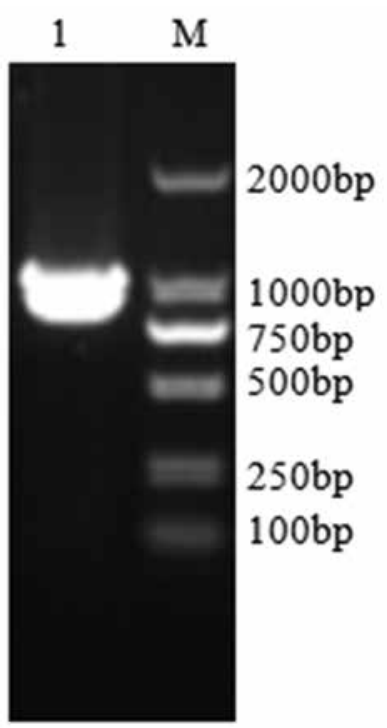

Figure 1. 5'RACE amplification results 1: Amplified product of 5'RACE PCR M: DL2000 Marker

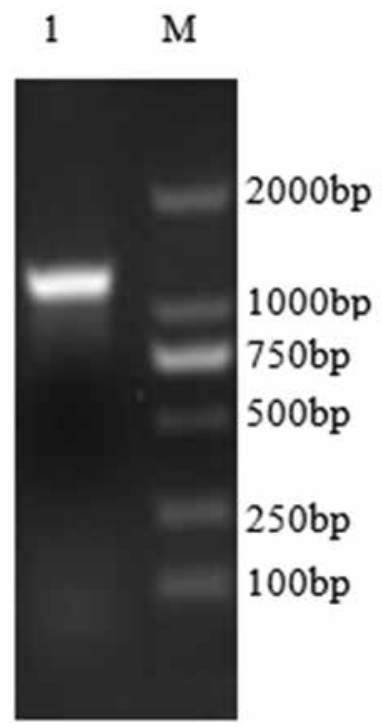

Figure 2. 3'RACE amplification results 1: Amplified product of 3'RACE PCR M: DL2000 Marker

ORF of 2721 nucleotides (Fig. S1*). The predicted protein encoded by the TaRPM1 gene consisted of 907 amino acids (Fig. S2) with a predicted isoelectric point of 4.86 .

\section{Characterization of the primary structure of the TaRPM1 homolog}

PROSITEScan analysis suggested that the TaRPM1 protein consists of five conserved domains: RX-CC_like, AAA_22, LRR_4, NB-ARC, and LRR_8. The NB-ARC domain was located at positions 175-454 aa, and the LRR subdomain of LRR_4 and LRR_ 8 was located at 594-633 aa and 594-645 aa, respectively.

The conserved region of the TaRPM1 homolog was compared with RPM1 regions from other plant species. The nucleotide sequences were highly conserved in RPMI genes, with sequence identities ranging from 75 to $99 \%$, including Aegilops tauschii at $99 \%$, Hordeum vulgare at $86 \%$, and Oryza sativa at $75 \%$. In addition, the predicted protein identities were compared with seven plant species: Aegilops tauschii at 98\%, Triticum urartu at 95\%, Hordeum vulgare at 79\%, Sorghum bicolor at 70\%, Oryza sativa Indica Group at 70\%, Brachypodium distachyon at $68 \%$ and Zea mays at $67 \%$.

The overall sequence alignment indicated that $R P M 1$ is relatively conserved among the seven plant species. Seven available $R P M 1$ sequences of various plant species were downloaded from GenBank and then employed to establish a neighbor-joining phylogenetic tree using MEGA software version 7.0 with 1000 bootstraps (Fig. 3). Results showed that the plant RPM1s can be separated into four main divisions. No transmembrane or

*Further details about the Electronic Supplementary Material (ESM) can be found at the end of the article. 


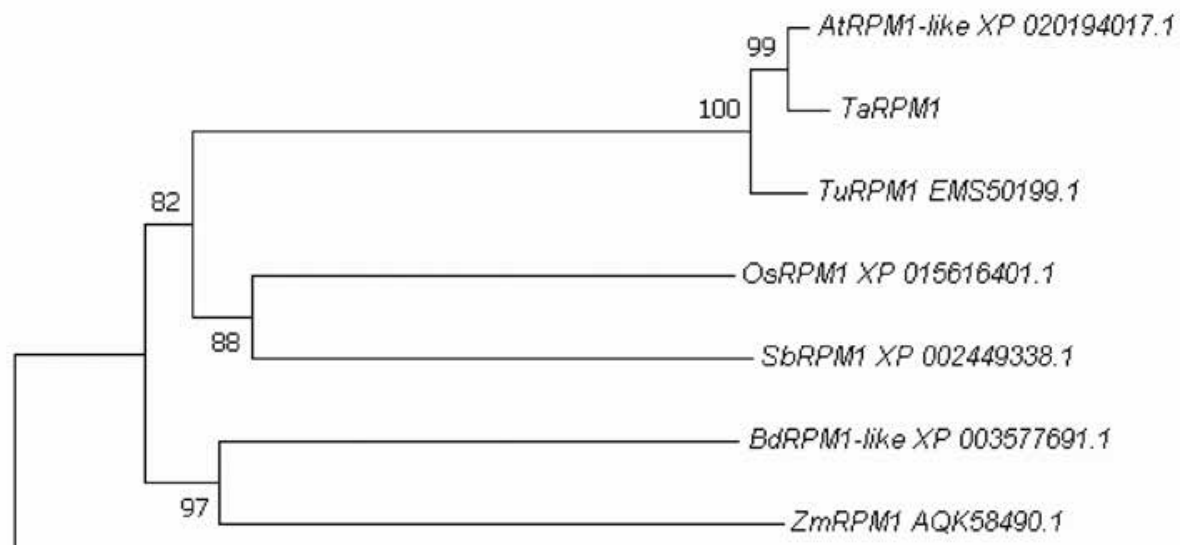

ObRPM1-like XP 006662833.1

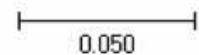

Figure 3. Neighbor-joining phylogenetic tree of whole RPM1 protein sequences from different species using MEGA 7.0 software. Branches are labeled with plant species names (At - Aegilops tauschii; $\mathrm{Sb}-$ Sorghum bicolor; $\mathrm{Tu}$ - Triticum urartu; $\mathrm{Bd}$ - Brachypodium distachyon; Os - Oryza sativa; $\mathrm{Zm}$ - Zea mays; $\mathrm{Ob}$ - Oryza brachyantha), followed by the gene name and GenBank accession number

signal peptide were confirmed by TMpred and Signal IP 3.0 analysis. Through location prediction using PSORT, TaRPM1 was found to be outside of cells with a probability of $67.1 \%$.

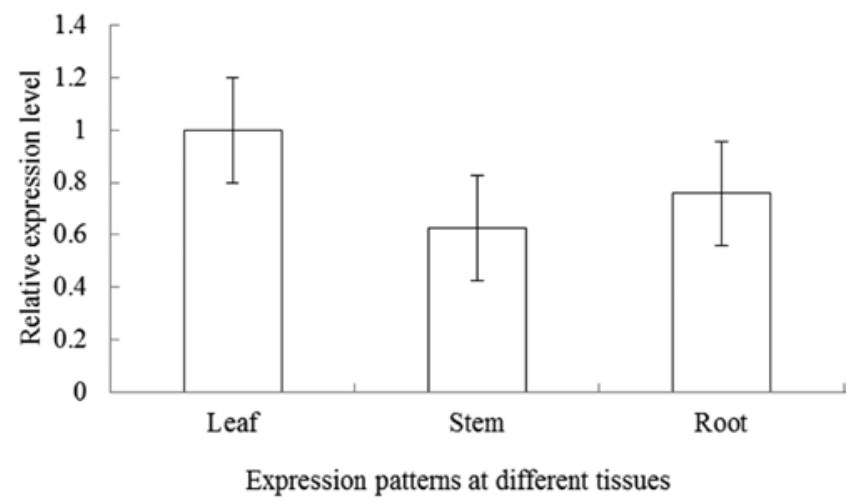

Figure 4. qRT-PCR analyses of the TaRPMI expression in wheat leaf, stem, and root tissues, and expression patterns of TaRPM1 in wheat leaves after inoculation with E09. Data were normalized to wheat tubulin expression level. The mean expression value was calculated with three independent replicates. Vertical bars represent the standard deviation 


\section{Spatial expression patterns of TaRPM1}

We next examined the transcription patterns of TaRPM1 in tissues, including root, stem and seedling leaves, in response to infection with powdery mildew E09 using qRT-PCR analyses (Fig. 4). The transcription of TaRPM1 in wheat leaves was higher than in both stem and root samples. Followed by the root, the stem samples had the lowest expression, which was approximately half of the expression level than that of the leaf samples. The expression of TaRPM1 in wheat roots, stems, and leaves was not significantly statistically different. We also analyzed the spatial tissues expression of TaRPM1 using Expression Atlas website. The result showed the expression level of RPMI in Arabidopsis leaves, roots were expression. There was also no significant difference in expression levels.

\section{Expression changes of TaRPM1 in response to Bgt infection}

Real-time PCR results showed that TaRPM1 expression increased between 0 to 48 hpi in an incompatible interaction (Fig. 5). The expression of TaRPM1 was up-regulated as early as 6 hpi. The highest level was reached at 48 hpi, showing a transcript level was approximately twenty-five-fold higher than the control. The level then slightly decreased to $72 \mathrm{hpi}$ and increased again to $96 \mathrm{hpi}$ and stayed at a high level until $120 \mathrm{hpi}$.

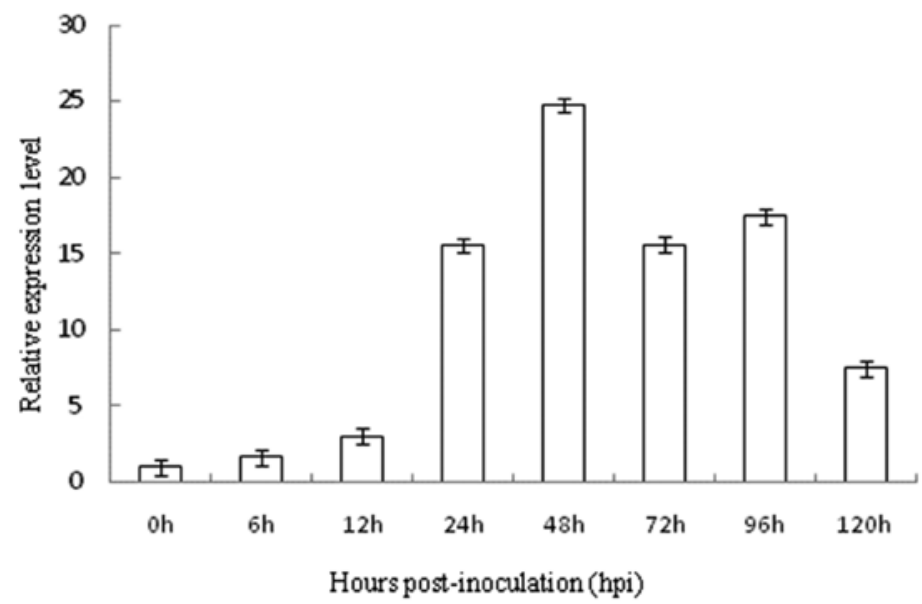

Figure 5. Expression patterns of the RPMI assessed by real-time PCR. Total RNA was isolated from young leaves of N9134 induced by powdery mildew infection at $0,6,12,24,48,72,96$, and 120 hpi. The a-tubulin genes were used as internal controls. Y-axis indicats the fold changes of miRNA expression in the stress samples compared with the mock samples. Data were averages of three independent biological experiments.

Standard deviation (SD) is shown as an error bar 


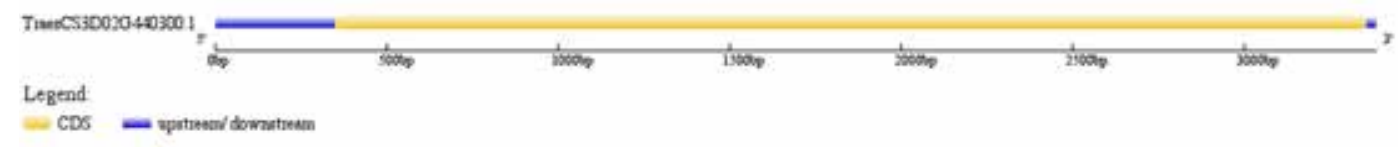

Figure 6. Structural prediction of TaRPMI using GSDS 2.0. The blue portion is the upstream or downstream sequence and the yellow is the coding sequence

In silico mapping of TaRPM1

The chromosome location of TaRPM1 was determined using in silico mapping analysis. By comparing the TaRPM1 gene with the latest sequencing data from Chinese Spring, the sequence similarity with $3 \mathrm{~A}, 3 \mathrm{~B}$ and $3 \mathrm{D}$ was $93.60 \%, 98.16 \%$, and $98.73 \%$, respectively. We also used the GSDS 2.0 for structural prediction of TaRPM1 that has a CDS domain (Fig. 6). Based on an analysis of sequence similarities, we inferred that the TaRPM1 gene is likely to be located on the wheat $3 \mathrm{D}$ chromosome.

\section{Discussion}

In this study, we described the isolation and characterization of the TaRPM1 gene from the wheat germplasm N9134 after inoculation with powdery mildew E09. The TaRPM1 gene was located on chromosome 3D. In addition, we also investigated the expression pattern of the TaRPMI in response to treatments with powdery mildew.

Most of the resistance proteins encoded by NBS-LRR disease-resistant genes are associated with plant disease resistance processes (Belkhadir et al. 2004). A resistance gene of TaRPM1 was cloned which, contained a complete ORF, consisting of 2721 nucleotides. Compared with the Chinese Spring sequence at 3D chromosome published by Appels et al. (2018), the sequence identity was $98.73 \%$. The differences in the sequence were mainly due to mutations at individual sites. The main cause of this phenomenon may be the difference in research materials. It may also be caused by the evolution of $\mathrm{R}$ genes, which is driven by selection on allelic variation created by mutation and reassortment (Hulbert et al. 2001). Sequence structure analysis showed that the TaRPM1 gene had a conserved region typical of a serine/threonine kinase structure. Studies have shown that NBS-LRR genes have evolved rapidly in bread wheat ( $\mathrm{Gu}$ et al. 2015). However, our phylogenetic analysis showed that the TaRPM1 gene was highly homologous in the plant at deduced amino acid levels. The results further suggest that the TaRPM1 gene evolved slowly in wheat.

The domain architecture of NBS-LRR was consistent with a role in pathogen recognition and defense response signaling (Amelinetorregrosa et al. 2008). In this study, the TaRPM1 gene did not contain a transmembrane structure and a signal peptide, suggesting that TaRPM1 is made of soluble cytoplasmic proteins. This finding is consistent with previous research results (Boyes et al. 1998).

Most $\mathrm{R}$ genes are constitutively expressed in the plant (Thurau et al. 2003). TaRPM1 was found to show no tissue-specific expression and to be regulated by powdery mildew, TaRPM1 gene expression was observed in roots, stems and leaves, respectively. Mean- 
while, the expression level of $R P M 1$ was also not significantly different in the leaves and roots of Arabidopsis using the Expression Atlas website. Some R genes were required for maintaining or enhancing the induction of signal transduction pathways, leading to resistance (Li et al. 2011). The psoRPM1 gene is capable of helping set up a resistance mechanism against $M$. incognita infection in tobacco ( $\mathrm{Li}$ et al. 2013). The expression of the psoRPM1 gene was highest 12 hours after inoculation with root-knot nematodes (Li et al. 2011). In this study, the RT-PCR results demonstrated that TaRPM1 expression was increased in N9134 after powdery mildew infection. The expression level of TaRPM1 was the highest at 48 hpi. Compared with N9134 results at 6 hpi, the expression level of TaRPM1 increased 8 times. The results of the study were inconsistent with those of Jiang et al. (2015). However, the results indicated that the expression level of the RPMI gene has a high expression level in disease resistant materials.

With the continuous improvement of wheat genome sequencing data, the silico mapping method has the advantages of simplicity and quickness compared with the traditional Chinese Spring deletion lines positioning method. In the wheat genome, 2406 sequences of NBS domain were isolated (Qiao et al. 2016), which is much higher than other monocotyledonous crops.

The sequences having the NBS sequence structure on the $3 \mathrm{~A}, 3 \mathrm{~B}$, and $3 \mathrm{D}$ chromosomes of wheat were 71, 133, and 71, respectively (Qiao et al. 2016). In our past research, the PmAS846 gene from wheat line N9134 was located on chromosome 5BL (Xue et al. 2012). Line N9134 is highly resistant to immunity against powdery mildew in Guanzhong, Shaanxi Province (Ji et al. 1999). In this study, however, TaRPM1 was located at chromosome 3D, and is apparently different than PmAS846. Due to TaRPM1 was highly expressed in wheat line N9134 after the powdery mildew inoculated. Therefore, we hypothesize that TaRPM1 is involved in PmAS846-mediated resistance to powdery mildew.

In summary, the TaRPM1 gene was isolated from the wheat germplasm N9134 and infected by powdery mildew. TaRPMI was expressed in root, stem and leave of wheat, and the difference in expression was not statistically significant. TaRPM1 had a strong response to powdery mildew at $48 \mathrm{hpi}$. In addition, TaRPM1 is located on the $3 \mathrm{D}$ arm of the wheat chromosome when using silico mapping. We found that TaRPM1 plays a significant role in response to powdery mildew infection.

\section{Acknowledgements}

This study was supported financially by the National Natural Science Foundation of China (No. 31460346), and the Corps scientific and technological research (2018AB040). Authors' contribution: The experiment was conceived and designed by W.Q. Ji, and was performed by Y.B. Nie. The data was analyzed by Y.B. Nie. The manuscript was written and edited by Y.B. Nie and W.Q. Ji. 


\section{References}

Amelinetorregrosa, C., Wang, B.B., O’Bleness, M.S., Deshpande, S., Zhu, H., Roe, B., Young, N.D., Cannon, S.B. 2008. Identification and characterization of nucleotide-binding site-leucine-rich repeat genes in the model plant Medicago truncatula. Plant. Physiol. 146:5-21.

Appels, R., Eversole, K., Feuillet, C. 2018. Shifting the limits in wheat research and breeding using a fully annotated reference genome. Science 361:Eaar 7191.

Belkhadir, Y., Nimchuk, Z., Hubert, D.A., Mackey, D., Dangl, J.L. 2004. Arabidopsis RIN4 negatively regulates disease resistance mediated by RPS2 and RPM1 downstream or independent of the NDR1 signal modulator and is not required for the virulence functions of bacterial type III effectors AvrRpt2 or AvrRpm1. Plant.cell. 16:2822-2835.

Boyes, D.C., Nam, J., Dangl, J.L. 1998. The Arabidopsis thaliana RPM1 disease resistance gene product is a peripheral plasma membrane protein that is degraded coincident with the hypersensitive response. Proc. Natl. Acad. Sci. USA. 95:15849-15854.

Cao, A., Xing. L., Wang, X., Yang, X., Wang, W., Sun, Y., Qian, C., Ni, J., Chen, Y., Liu, D., Wang, X., Chen, P. 2011. Serine/threonine kinase gene $\operatorname{Stpk}-V$, a key member of powdery mildew resistance gene $P m 21$, confers powdery mildew resistance in wheat. Proc. Natl. Acad. Sci. USA. 108:7727-7732.

Collins, N., Park, R.W., Ellis, J., Pryor, A.J. 2001. Resistance gene analogs in barley and their relationship to rustresistance genes. Genome 44:375-381.

Gong, C., Cao, S., Fan, R., Wei, B., Chen, G., Wang, X., Li, Y., Zhang, X. 2013. Identification and phylogenetic analysis of a CC-NBS-LRR encoding gene assigned on chromosome 7B of wheat. Int. J. Mol. Sci. 14:15330-15347.

Grant, M.R., Godiard, L., Straube, E., Ashfield, T., Lewald, J., Sattler, A., Innes, R.W., Dang, J.L. 1995. Structure of the Arabidopsis RPM1 gene enabling dual specificity disease resistance. Science 269:843-846.

Gu, L., Si, W., Zhao, L., Yang, S., Zhang, X. 2015. Dynamic evolution of NBS-LRR genes in bread wheat and its progenitors. Mol. Genet. Genomic. 290:727-738.

Hulbert, S.H., Webb, C.A., Smith, S.M., Sun, Q. 2001. Resistance gene complexes: evolution and utilization. Annu. Rev. Phytopathol. 39:285-312.

Ji, W.Q., Xue, X.Z., Wang, Q.Y., Wang, C.Y., Zhao, H.X., Zhang, H. 1999. Transfer of the powdery mildew resistant gene from $T$. dicoccoides into T. aestivum and its RAPD analysis. Acta Bot. Bor.Occid. Sin. 19:31-36.

Jiang, L.Y., Liu, J., Liu, X.Y., Wang, Z.Y. 2015. Cloning and functional verification of wheat resistant related gene TaRPM1 against powdery mildew. In: The 6th wheat genomics and molecular breeding conference, Yanglin, Shaanxi, China. pp. 86.

Li, F., Zhou, L., Xiao, F., Liao, K., Hu, J. 2011. Characterization of the psoRPM1 gene for resistance to rootknot nematodes in wild myrobalan plum (Prunus sogdiana). Afr. J. Biotechnol. 10:12859-12867.

Li, F., Zhu, X., Qiao, F., Chen, X.F., Li, H., Hu, J.F. 2013. psoRPM1 Gene from Prunus sogdiana Indicated Resistance to Root-knot Nematode in Tobacco. Acta. Horti. Sinica. 40:2497-2504.

McHale, L., Tan, X., Koehl, P., Michelmore, R.W. 2006. Plant NBS-LRR proteins: adaptable guards. Genome. Biol. 7:1-11.

Periyannan, S., Moore, J., Ayliffe, M., Bansal, U., Wang, X., Huang, L., Deal, K., Luo, M., Kong, X., Bariana, H., Mago, R., McIntosh, R., Dodds, P., Dvorak, J., Lagudah, E. 2013. The gene Sr33, an ortholog of barley Mla genes, encodes resistance to wheat stem rust race Ug99. Science 341:786-788.

Qiao, L.Y., Chang, J.Z., Guo, H.J., Gao, J.G., Zheng, J., Chang, Z.J. 2016. Genome-wide analysis of TaNBS resistance genes and development of chromosome 2AL-specific NBS-SSR markers in wheat. Acta Agron. Sinica. 42:795-802.

Saintenac, C., Zhang, W., Salcedo, A., Rouse, M.N., Trick, H.N., Akhunov, E., Dubcovsky. J. 2013. Identification of wheat gene Sr35 that confers resistance to Ug99 stem rust race group. Science 341:783786.

Srichumpa, P., Brunner, S., Keller, B., Yahiaoui, N. 2005. Allelic series of four powdery mildew resistance genes at the Pm3 locus in hexaploid bread wheat. Plant. Physiol. 139:885-895. 
Swiderski, M.R., Birker, D., Jones, J.D.G. 2009. The TIR domain of TIR-NB-LRR resistance proteins is a signaling domain involved in cell death induction. Mol. Plant. Microbe. In. 22:157-165.

Thurau, T., Kifle, S., Jung, C., Cai, D. 2003. The promoter of the nematode resistance gene Hs $1^{\text {pro-1 }}$ activates a nematode-responsive and feeding site-specific gene expression in sugar beet (Beta vulgaris L.) and Arabidopsis thaliana. Plant. Mol. Biol. 52:643-660.

Tommasini, L., Yahiaoui, N., Srichumpa, P., Keller, B. 2006. Development of functional markers specific for seven Pm3 resistance alleles and their validation in the bread wheat gene pool. Theor. Appl. Genet. 114:165-175.

Wang, C.Y., Ji, W.Q., Zhang, G.S., Wang, Q.Y., Cai, D.M., Xue, X.Z. 2007. SSR markers and preliminary chromosomal location of a powdery mildew resistance gene in common wheat germplasm N9134. Acta Agron. Sin. 33:163-166.

Xue, F. 2012. Molecular mapping of the powdery mildew resistance gene and transcriptome analysis of the wheat-blumeria graminis f. sp. tritici interaction. PhD thesis. College of Agronomy, Northwest A\&F University. Yangling, China.

Xue, F., Ji, W., Wang, C., Zhang, H., Yang, B. 2012. High-density mapping and marker development for the powdery mildew resistance gene PmAS846 derived from wild emmer wheat (Triticum turgidum var. dicoccoides). Theor. Appl. Genet. 124:1549-1560.

Yahiaoui, N., Srichumpa, P., Dudler, R., Keller, B. 2004. Genome analysis at different ploidy levels allows cloning of the powdery mildew resistance gene $P m 3 b$ from hexaploid wheat. Plant. J. 37:528-538.

\section{Electronic Supplementary Material (ESM)}

Electronic Supplementary Material (ESM) associated with this article can be found at the website of CRC at https://akademiai.com/loi/0806

Electronic Supplementary Figure S1. TaRPM1 gene sequence

Electronic Supplementary Figure S2. Protein sequence 